University of New Hampshire

University of New Hampshire Scholars' Repository

\title{
CdZnTe strip detectors as sub-millimeter resolution imaging gamma radiation spectrometers
}

\author{
K Larson \\ University of New Hampshire - Main Campus \\ V Boykin \\ University of New Hampshire - Main Campus \\ M L. Cherry \\ Louisiana State University - Baton Rouge \\ J F. Courville \\ University of Montreal \\ F P. Doty \\ DIGIRAD
}

See next page for additional authors

Follow this and additional works at: https://scholars.unh.edu/ssc

Part of the Astrophysics and Astronomy Commons

\section{Recommended Citation \\ Larson, K.; Boykin, D.V.; Cherry, M.L.; Courville, J.F.; Doty, F.P.; Drake, A.; Guzik, T.G.; Hamel, L.A.; Macri, J.R.; Mayer, M.; McConnell, M.L.; Ryan, J.M.; Tousignant, O., "CdZnTe strip detectors as sub-millimeter resolution imaging gamma radiation spectrometers," Bioengineering Conference, 1997., Proceedings of the IEEE 1997 23rd Northeast, vol., no., pp.59,60, 21-22 May 1997.}

This Conference Proceeding is brought to you for free and open access by the Institute for the Study of Earth, Oceans, and Space (EOS) at University of New Hampshire Scholars' Repository. It has been accepted for inclusion in Space Science Center by an authorized administrator of University of New Hampshire Scholars' Repository. For more information, please contact Scholarly.Communication@unh.edu. 


\section{Authors}

K Larson, V Boykin, M L. Cherry, J F. Courville, F P. Doty, A Drake, T G. Guzik, L A. Hamel, John R. Macri, M Mayer, Mark L. McConnell, James M. Ryan, and O Tousignant 


\title{
CdZnTe Strip Detectors as Sub-millimeter Resolution Imaging Gamma Radiation Spectrometers
}

\author{
K. Larson 1 , D.V. Boykin 1 , M.L. Cherry ${ }^{3}$, J.F. Courville ${ }^{2}$, \\ F.P. Doty ${ }^{4}$, A. Drake 1 , T.G. Guzik ${ }^{3}$, L.A. Hamel ${ }^{2}$, \\ J.R. Macri ${ }^{1}$, M. Mayer ${ }^{1}$ M.L. McConnell 1 , J.M. Ryan ${ }^{1}$, O. Tousignant ${ }^{2}$ \\ ${ }^{1}$ Space Science Center, University of New Hampshire, Durham, NH 03824 USA \\ 2 Department of Physics, University of Montreal, Montreal, H3C 3J7, Canada \\ 3 Department of Physics and Astronomy, Louisiana State University, Baton Rouge, LA 70803 USA \\ 4 DIGIRAD, San Diego, CA 92121-2410 USA
}

\begin{abstract}
We report $\gamma$-ray detection performance measurements and computer simulations of a sub-millimeter pitch $\mathrm{CdZnTe}$ strip detector. The detector is a prototye for $\gamma$-ray measuremets in the range of $20-600 \mathrm{keV}$. The prototype is a $1.5 \mathrm{~mm}$ thick, 64 $\times 64$ orthogonal stripe $\mathrm{CdZnTe}$ detector of $0.375 \mathrm{~mm}$ pitch in both dimensions, with approximately one square inch of sensitive area. Using discrete laboratory electronics to process signals from an $8 \times 8$ stripe region of the prototype we measured good spectroscopic uniformity and sub-pitch $(\sim 0.2$ $\mathrm{mm}$ ) spatial resolution in both $x$ and $y$ dimensions. We present below measurements of the spatial uniformity, relative timing and pulse height of the anode and cathode signals. We simulated the photon interactions and signal generation in the strip detector and the test electronics and we compare these results with the data. The data indicate that cathode signal-as well as the anode signal-arises more strongly from the conduction electrons rather than the holes.
\end{abstract}

\section{INTRODUCTION}

Sub-millimeter resolution CdZnTe position-sensitive detector technology is a strong prospect for achieving both improved energy and spatial resolution with high stopping power and without the need for cryogenic cooling $[1,2,3,4$, $5,6]$. The operating principles of $\mathrm{CdZnTe}$ strip detectors are described in ref. [4]. A major goal of the efforts is the development of large area imaging modules incorporating strip detectors and the associated electronics in compact packages. These modules could be incorporated in PET or SPECT devices, which currently have a spatial resolution of 3-4 mm FWHM. [11]

Demonstrations of the spectroscopic and imaging properties of prototype $\mathrm{CdZnTe}$ strip detectors have been a focus of much of this work [1, 2, 4]. Modeling of the photon interaction, charge transport and signal generation in these detectors is also important $[7,8,9,10]$. Achieving agreement between simulations and prototype measurements will be a significant development milestone. Accurate modeling will help specify optimum detector geometries and appropriate signal processing electronics, thus minimizing the number of expensive and time consuming hardware prototypes.

\section{DESCRIPTION AND TEST SETUP}

The prototype detector was manufactured by DIGIRAD [1]. A pattern of $64 \times 64$ interdigitated and orthogonal contact stripes on each surface defines a $24 \mathrm{~mm} \times 24 \mathrm{~mm}$ imaging area $\left(5.76 \mathrm{~cm}^{2}\right)$ on a $28 \mathrm{~mm} \times 28 \mathrm{~mm} \mathrm{CdZnTe}$ substrate that is $1.5 \mathrm{~mm}$ thick. The stripe pitch is $0.375 \mathrm{~mm}$ with a 0.15 mm gap between stripe contacts.

Figure 1 illustrates the laboratory setup for the prototype strip detector measurements. Independent ac-coupled signal channels for each of 8 consecutive stripes in each dimension define a $3 \mathrm{~mm} \times 3 \mathrm{~mm}$ active test region of 64 "pixels," i.e. $1 / 64$ of the detector's area. Amptek A225 preamp/shaper circuits and additional op amps provide fast (200 ns rise time) signals for level discrimination and coincidence logic and slow $(2 \mu \mathrm{s})$ channels for pulse height measurements on each stripe. All stripes on each detector surface are biased to assure a uniform electric field in the CdZnTe. The load resistance is 1 $\mathrm{G} \Omega$. Any $x-y$ discriminator coincidence triggers the readout of 17 parameters for each event: 16 pulse heights and the relative arrival time of coincident anode and cathode signals. The typical bias used for these measurements is $200 \mathrm{~V}$. All measurements were performed at room temperature.

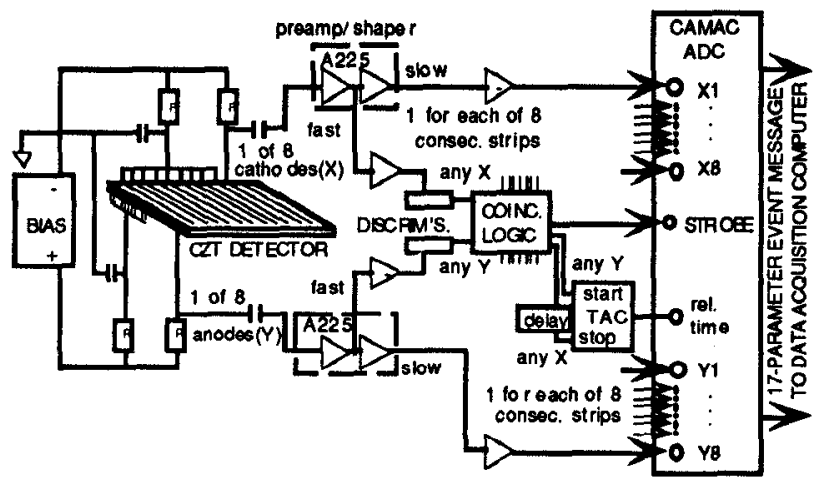

Figure 1. Test setup (orthogonal stripe coincidence mode)

The simulation model is intended to be an end-to-end tool for simulating all detection and measurement processes from photon interaction to the electronics. The detector material (mobilities, trapping and detrapping coefficients), bias, and geometry (thickness, electrode pitch and gap) of the prototype detector are represented in the model. A GEANT module 
simulates the photon interaction locations, the energy deposit and the distribution of the ionization charges for incident photons of any given energy. A charge transport and signal generation module $[7,9,10]$ computes the signal induced on any electrode for photon interaction locations at any depth $z$ and any lateral $(x, y)$ position in the detector.

TEST AND SIMULATION RESULTS

It has been shown that signal charge for each detected event is shared by the triggering anode stripe and, at most, one, of its neighbors [1]. The charge transport and signal generation simulations support the observation that the event is mostly sensed by the two nearest electrodes on each plane, though, as previously mentioned, small signals are induced in farther electrodes. That effect is not a result of carrier diffusion but of induced current through various electrodes by carriers drifting in the detector and it strongly depends on the electronics integration time.

Figure 2a shows the response of a single "pixel" of the prototype detector to $122 \mathrm{keV}$ photons from a ${ }^{57} \mathrm{Co}$ source. The GEANT model also predicts nearly total energy deposit for the 122 and $136 \mathrm{keV}$ photons of ${ }^{57} \mathrm{Co}$ with a fraction of fluorescence escape events similar to that measured. Figure $2 b$ is the sum of the anode histograms from all 64 pixels and illustrates the good uniformity of response across the $8 \times 8$ stripe test region.

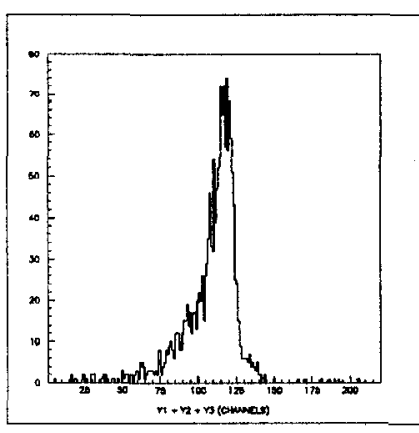

Fig. 2a. $122 \mathrm{keV}$ single "pixel" spectrum

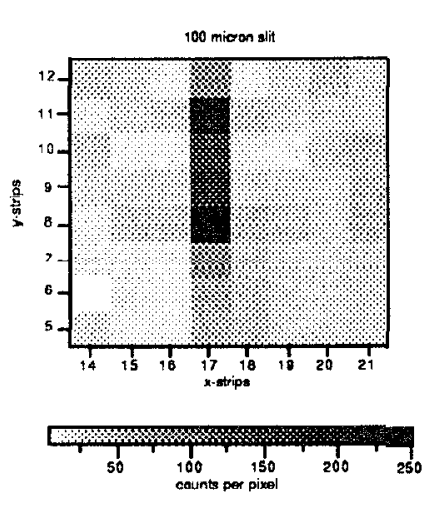

Fig. 3a. Image produced by a $100 \mu \mathrm{m}$ wide slit.

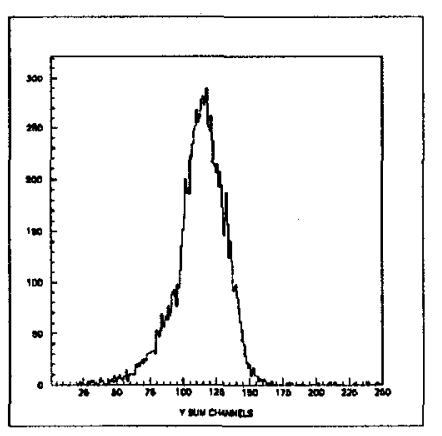

Fig. 2b. $122 \mathrm{keV}$ sum spectrum of 64 "pixels."

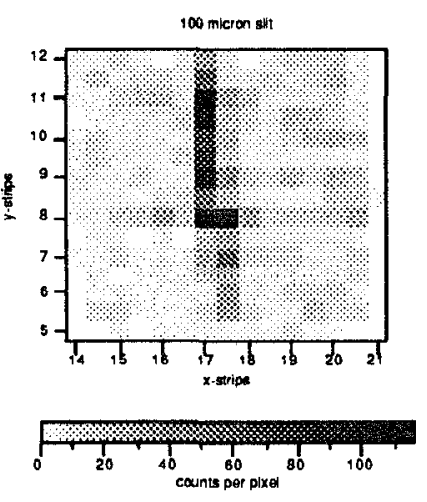

Fig. $3 b .100 \mu \mathrm{m}$ wide slit using half-pixels. Note slit tilt relative to $y$-axis
Figures $3 \mathrm{a}$ and $3 \mathrm{~b}$ are images obtained from the $8 \times 8$ stripe $\left(3 \times 3 \mathrm{~mm}^{2}\right)$ test region of the prototype using $122 \mathrm{keV}$ photons. In figure $5 \mathrm{a}$ event location is assigned to the stripe in each dimension recording the highest pulse height $(0.375 \mathrm{~mm}$ pixels in each dimension). In figure $5 b$ event location is assigned based on interpolation of the pulse heights recorded by neighboring stripes (two pixels per stripe pitch, $\sim 0.19$ $\mathrm{mm}$ pixels in each dimension).

The slit images also illustrate the non-uniformity of event location response, with the count rate of neighboring pixels differing by as much as a factor of two. Small bias differences between stripes may be responsible for some if not all of the non-uniformity. We have shown [11] that even a one Volt change in the differential bias can result in a trigger rate change of $>30 \%$, with neighboring stripes showing a complimentary change.

A decrease is observed in both the coincident trigger rate and the signal pulse height when the detector is illuminated from the anode side instead of the cathode side. This effect is most evident with lower energy photons having a mean free path less than the detector thickness $(1.5 \mathrm{~mm})$. This indicates that events occouring near the anode side of the detector are less likely to trigger a coincidence. Anode pulse heights are relatively unaffected.

The measured relative arrival times of typical anode and cathode signals do not differ by more than 300 nsecs. A sample of coincident events observed directly from the pre-amp output with a digital oscilliscope shows that the simultaneity of anode and cathode signals is irrespective of pulse height or shape. The simulation predicts a delay in the arrival of holes at the cathode by as much as 2 microseconds due to poor hole mobiliy, but this is not observed in laboratory tests.

$$
\text { DISCUSSION }
$$

Sub-pitch spatial resolution( 190 um) in two dimensions and good energy resolution and spectral response have been demonstrated with this prototype. The spatial response is, however, non-uniform, but could be improved with a more careful biasing scheme. The simulation agrees qualitatively with many observations and trends seen in the data, but quantitatively predicts a larger component of the hole signal than is observed. Plans for the future include further measurements of CdZnTe transport properties in order to help resolve differences between the simulation and data, as well as exploring methods of compensating for poor hole collection in order to improve the spectral resolution.

[1-10] M. Mayer et al., "Performance and Simulation of CdZnTe Strip Detectors as Sub-millimeter Resolution Imaging Gamma Radiation Spectrometers," as submitted to IEEE Trans. Nucl. Sci., 25 February 1997

[11] K. Weinhard, M. Dahlbom, L. Ericksson, et al., "The ECAT EXACT HR: Performance of a new high resolution positron scanner", J. Comput. Assist. Tomog., vol 18, pp.110-118, 1994 